\title{
EDITORIAL
}

\section{The pleura: the outer space of pulmonary medicine}

\author{
H. Hamm, R.W. Light*
}

In this issue of the Journal, MiserocCHI [1] starts our series of articles on the pleura with a review of the physiology and the pathophysiology of the pleural space. In forthcoming months this series will cover a wide range of issues on pleural diseases. We have seen much progress in basic research as well as changing standards in the diagnosis and management of pleural disorders in recent years. The aim of this series is to supply the readership of the Journal with a concise and up-to-date overview on these developments, bringing some of the important information from the "outer space" of research down to the earth of everyday clinical practice.

The understanding of the physiology of the pleural space has come a long way since the pioneering work by scientists, such as Starling [2,3] at the end of the last century and VON NEERGARD [4] in the 1920s, who developed pressure control models of pleural fluid turnover. The relevant pressures were identified as the pleural liquid pressure (resulting from the expanding force of the thoracic cage versus the recoil force of the lung), the capillary and oncotic pressures in both the visceral and parietal membranes, and the oncotic pressure of the pleural fluid itself. The early models initially suggested a pressure-driven fluid flow from the parietal side across the pleural space to the visceral pleura and subsequently, into the pulmonary interstitium. Such models were derived from studies of animals with a thin visceral membrane, in which the blood supply originates from branches of the pulmonary arteries (with low capillary pressures). Later, it was found that in some other mammals, including man, the visceral pleura is considerably thicker and the blood supply is from the systemic circulation (with higher capillary pressures). This means that once the fluid enters the pleural space, there is no clear local pressure gradient to explain its further distribution. Thus, pressure models in man fall short in explaining the existence of a constant physiological amount of fluid in the pleural cavity and also, they do not give a clue as to the further fate of the fluid. In other words, volume control mechanisms need to be involved in addition to pressures.

Today, we know that under normal conditions in humans, the physiological pleural fluid is formed in apical parts of the parietal pleura and is drained by lymphatic stomata [5] which seem to be exclusively found on the parietal side and are most numerous in mediastinal and diaphragmatic areas. Thus, normal fluid ex-

Correspondence: H. Hamm, Abt. Pneumologie, Medizinische Universitätsklinik, D-79106 Freiburg, Germany. *University of California, Irvine Chief, Pulmonary Exercise Laboratory, Veterans Administration Medical Center, Long Beach, California, 90822, USA. change is dominated by the parietal side. Furthermore, there is probably a constant fluid flow from apical regions to mediastinal and diaphragmatic areas within the pleural cavity. It seems likely that there is very little if any relevant fluid exchange on the visceral side under normal conditions [6]. Furthermore, the parietal stomata posses valve-like structures to guarantee a unidirectional flow of fluid out of the pleural space. They may also have a role in maintaining a constant amount of fluid in the pleural space, which means they probably play a crucial role in volume control. However, the paper by MiserocCHI [1] expertly demonstrates how difficult it still is to understand the complex interrelationships of pleural pressures, fluid flows and anatomical structures. Furthermore, it gives us an idea of the work that is needed to clarify the physiological and pathophysiological mechanisms in detail.

Looking at the recent physiological and pathophysiological work, what are the lessons to learn for the clinician? One of the messages is that pulmonary hypertension or right heart failure alone do not lead to the accumulation of pleural fluid, in spite of persisting statements in may clinical and radiological textbooks that effusions associated with congestive heart failure reflect right heart failure. Experienced clinicians have long questioned this, since it is very unusual for patients with pure cor pulmonale or right heart failure to develop pleural effusions. Furthermore, recent work has shown that cardiogenic pleural effusions are closely related to elevated pulmonary capillary wedge pressures rather than to pulmonary artery or right heart pressures [6]. Thus, cardiogenic pleural effusions are usually related to left ventricular dysfunction with pathological flow of interstitial pulmonary fluid into the pleural space across the visceral side. In animal models of cardiogenic pulmonary oedema, it has been shown that up to $25 \%$ of the oedema fluid exits the lungs via the visceral pleura into the pleural space [7]. Removal of this fluid by thoracentesis reduces systemic fluid overload and should contribute to a decrease in pulmonary capillary pressures. It is therefore rational to include a therapeutic thoracentesis in the treatment repertoire of congestive heart failure whenever there is a significant pleural effusion.

One of the other findings that may be of interest to the clinician is that the reserve capacity of the pleural space to clear extra fluid is probably quite substantial. Although we are still lacking direct evidence from the human pleural space, it seems probable from animal studies that the lymphatic drainage system can cope with up to several hundred millilitres of additional fluid per day without the development of an effusion. Therefore, 
any pleural effusion represents a severe imbalance of pleural fluid formation and/or drainage capacity. In case of congestive heart failure, even a small pleural effusion signals a severe pulmonary fluid overload. On the other hand, the absence of effusions does not necessarily mean that the pleural drainage system is not already under severe stress.

A very interesting question is whether an intact pleural space has any useful function. Surprisingly, we do not have a convincing answer to this. Traditionally, it was thought that pleural symphysis should lead to a loss of lung function or at least to regional imbalances of ventilation. However, there is no evidence to suggest that lung function is significantly impaired in pleural symphysis and the number of imbalances of ventilation that have been described are negligible [8]. In elephants (a very successful species until man came around) the pleural space seems to be obliterated $[9,10]$, perhaps by a kind of "congenital pleurodesis". Considering the size of an elephant's pleural cavity and the fact that the pleural liquid pressure becomes more negative with increasing body mass (for details, see MisEROCCHI [1], in this issue), it is likely that pleural effusions or pneumothoraces would pose a severe threat to these animals. Thus, the evolutionary advantage of this phenomenon seems obvious: such species will not have to worry about pleural effusions or pneumothoraces. So, after all, what is the pleural space good for? It has been hypothesized that it could function as a "drip pan" for pulmonary oedema fluid. We have shown above that it can indeed play such a role, but is it an important function? Decades have passed since the introduction of pleurodesis and we are still unable to find reports of subsequent complicating pulmonary oedema [11], so we conclude that even this role is unlikely to be of clinical relevance.

Maybe the best argument, then, for the evolutionary survival of the pleural space in man is its convenience to surgeons in lung operations. Most thoracic surgical procedures would be very difficult to perform if the human pleural space had taken the evolutionary path of the elephant. It remains a philosophical question if this was really anticipated by Mother Nature or if the survival of the pleural space in man is rather a case of pure luck.

\section{References}

1. Miserocchi G. Physiology and pathophysiology of pleural fluid turnover. Eur Respir J 1997; 1: 219-225.

2. Starling EH, Tubby AH. On absorption from and secretion into the serous cavities. J Physiol (London) 1894; 16: $140-155$.

3. Starling EH. On the absorption of fluids from the connective tissue spaces. J Appl Physiol 1896; 19: 312-326.

4. von Neergard K. Zur Frage des Druckes im Pleuraspalt. Beitr Klin Erforsch Tuberk Lungenkr 1927; 65: 476485.

5. Gaudio E, Rendina EA, Pannarale L, Ricci C, Marinozzi G. Surface Morphology of the Human Pleura - A scanning electron microscopic study. Chest 1988; 92: 149152.

6. Wiener-Kronish JP, Broaddus VC. Interrelationship of pleural and pulmonary interstitial liquid. Ann Rev Physiol 1993; 55: 209-226.

7. Broaddus VC, Wiener-Kronish JP, Staub NC. Clearance of lung edema into the pleural space of volume loaded anesthetized sheep. J Appl Physiol 1990; 68: $2623-$ 2630.

8. Fleetham JA, Forkert L, Clarke H, Anthonisen NR. Regional lung function in the presence of pleural symphysis. Am Rev Respir Dis 1980; 122: 33-38.

9. Boas JEV. Fehlen der Pleurahöhlen beim indischen Elefanten. Morphol Jahrb 1906; 35: 494-495.

10. Agostoni E. Mechanics of the pleural space. Physiol Rev 1972; 52: 57-128.

11. Light RW. Pleural diseases (3rd.) Baltimore, Williams \& Wilkins, 1995. 